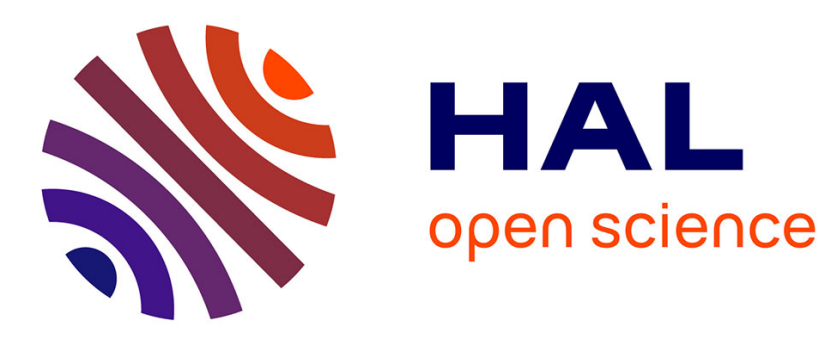

\title{
Étude d'un générateur à compression de champ magnétique utilisant un explosif gazeux
}

R. Hahn, B. Antoni, J. Lucidarme, C. Rioux, F. Rioux-Damidau

\section{To cite this version:}

R. Hahn, B. Antoni, J. Lucidarme, C. Rioux, F. Rioux-Damidau. Étude d'un générateur à compression de champ magnétique utilisant un explosif gazeux. Revue de Physique Appliquée, 1976, 11 (3), pp.409419. 10.1051/rphysap:01976001103040900 . jpa-00244076

\section{HAL Id: jpa-00244076 https://hal.science/jpa-00244076}

Submitted on 1 Jan 1976

HAL is a multi-disciplinary open access archive for the deposit and dissemination of scientific research documents, whether they are published or not. The documents may come from teaching and research institutions in France or abroad, or from public or private research centers.
L'archive ouverte pluridisciplinaire HAL, est destinée au dépôt et à la diffusion de documents scientifiques de niveau recherche, publiés ou non, émanant des établissements d'enseignement et de recherche français ou étrangers, des laboratoires publics ou privés. 


\title{
ÉTUDE D'UN GÉNÉRATEUR \\ A COMPRESSION DE CHAMP MAGNÉTIQUE UTILISANT UN EXPLOSIF GAZEUX
}

\author{
R. HAHN, B. ANTONI (*), J. LUCIDARME, C. RIOUX et F. RIOUX-DAMIDAU \\ Laboratoire de physique des plasmas, Groupe électrotechnique et fusion contrôlée, \\ Université de Paris XI, 91405 Orsay, France
}

(Reçu le 2 octobre 1975, accepté le 22 janvier 1976)

\begin{abstract}
Résumé. - On décrit un prototype expérimental de générateur à compression de champ magnétique dans lequel l'explosif solide habituel est remplacé par le mélange $2 \mathrm{H}_{2}+\mathrm{O}_{2}$ à la pression de 60 bars.

La partie conductrice est coaxiale ; elle est formée d'un tube d'aluminium contenant le mélange et mis dans une carcasse conductrice. Un fil explosant placé le long de l'axe du tube déclenche l'explosion qui accélère le tube radialement vers l'extérieur. Le champ magnétique créé initialement dans la structure coaxiale par la décharge d'un banc de condensateurs est alors comprimé par le tube. Partant d'un courant initial de $0,8 \mathrm{MA}$ nous avons obtenu 4,75 MA, l'énergie passant de 10 à $30 \mathrm{~kJ}$.

Dans ce type de générateurs, seul le tube est détruit et des énergies supérieures à $10 \mathrm{MJ}$ peuvent être facilement obtenues à un prix très bas et avec un bon rendement chimique. Le mélange $2 \mathrm{H}_{2}+\mathrm{O}_{2}$ est produit par électrolyse, ce qui évite le stockage d'explosifs à haute énergie.
\end{abstract}

Abstract. - We present a preliminary experimental compression generator for which the normal solid explosive is replaced by a gaseous mixture $2 \mathrm{H}_{2}+\mathrm{O}_{2}$ at a pressure of 60 bars. The mixture is contained in a coaxial, conducting structure in the form of an aluminium tube which is placed in a conducting carcass. An exploding wire placed axially in the tube initiates the explosion which accelerates the tube radially outwards. The magnetic field initially created by the discharge of a capacitor bank through the coaxial structure is thus compressed by the tube. With an initial current of $0.8 \mathrm{MA}$, we have obtained $4.75 \mathrm{MA}$, the energy increasing from 10 to $30 \mathrm{~kJ}$.

With this type of generator, only the tube is destroyed and energies greater than $10 \mathrm{MJ}$ can be delivered without undue difficulty for a minimum of total cost and with a good chemical efficiency. The mixture $2 \mathrm{H}_{2}+\mathrm{O}_{2}$ is produced by electrolysis, avoiding the storage of high energy explosives.

1. Introduction. - Les techniques de production d'énergies magnétiques élevées (quelques mégajoules en quelques centaines de microsecondes) par les générateurs à compression de champ magnétique sont actuellement assez bien connues [1,6]. Ces générateurs, qui utilisent tous jusqu'à présent de l'explosif solide, permettent de réaliser des expériences de plasma tout comme les bancs de condensateurs rapides d'énergie élevée, mais avec une installation nettement moins onéreuse [7]. Cependant, les destructions violentes que ce type d'explosif entraîne font obstacle à la généralisation de l'emploi de ces générateurs ; ils ne sont utilisables que par les laboratoires disposant d'abris et de champs de tirs spécialement équipés.

Cet article propose et montre la possibilité de réaliser un générateur à compression de champ magnétique particulièrement peu onéreux, d'un fonctionne-

$\left(^{*}\right)$ Commissariat à l'Energie Atomique. Centre d'Etude de Limeil, B. P. 27, 94190 Villeneuve-Saint-Georges, France. ment très peu destructif et par suite utilisable à l'intérieur même d'un laboratoire conventionnel [8]. Il emploie un explosif peu brisant : le mélange $2 \mathrm{H}_{2}+\mathrm{O}_{2}$ sous une pression de 60 bars qui est, dans ces conditions, suffisamment doux pour que les destructions soient limitées aux seules pièces en mouvement, mais suffisamment puissant pour que les performances soient finalement peu différentes de celles des générateurs à explosif solide. Nous décrirons ici un montage d'essai et les résultats obtenus qui montrent la faisabilité de ce nouveau type de générateurs.

2. Principe de fonctionnement. - Les générateurs à compression de champ magnétique sont des systèmes dont l'énergie électrique initiale est accrue par la conversion de l'énergie chimique d'un explosif résultant d'une déformation rapide qui réduit l'inductance du circuit électrique. Ils peuvent être schématisés par un circuit comprenant en série une inductance $L(t)$ et une résistance $R(t)$ temporellement variables (cf. Fig. 1). 


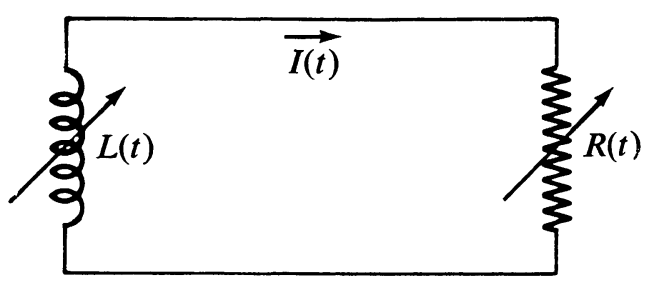

FIG. 1.

A l'instant initial le circuit est parcouru par un courant $I_{0}$ et l'énergie magnétique emmagasinée dans l'inductance est $W_{0}=\frac{1}{2} L_{0} I_{0}^{2}$.

L'équation électrique du circuit est :

$$
\frac{\mathrm{d}}{\mathrm{d} t}(L I)+R I=0
$$

dont la solution peut s'exprimer par

$$
L I=L_{0} I_{0} \exp -\int_{0}^{t} \frac{R(t)}{L(t)} \mathrm{d} t .
$$

Nous appellerons coefficient de conservation du flux

$$
\lambda(t)=\exp -\int_{0}^{t} \frac{R}{L} \mathrm{~d} t
$$

et taux de compression sans pertes le rapport

$$
\Gamma(t)=\frac{L_{0}}{L(t)} .
$$

Avec ces notations la multiplication du courant et de l'énergie magnétique s'écrivent :

$$
\begin{aligned}
\frac{I(t)}{I_{0}} & =\Gamma \lambda(t) \\
\frac{W}{W_{0}} & =\Gamma \lambda^{2}(t) .
\end{aligned}
$$

En multipliant l'éq. (1) par $I(t)$, nous obtenons l'équation d'évolution de l'énergie magnétique du circuit :

$$
\frac{\mathrm{d}}{\mathrm{d} t}\left(\frac{1}{2} L I^{2}\right)+R I^{2}=-\frac{1}{2} I^{2} \frac{\mathrm{d} L}{\mathrm{~d} t} .
$$

Elle montre que la puissance fournie $-\frac{1}{2} I^{2} \frac{\mathrm{d} L}{\mathrm{~d} t}$ pour diminuer l'inductance accroît l'énergie magnétique du circuit tant que $-\mathrm{d} L / \mathrm{d} t>2 R$ cependant qu'une partie est perdue en effet Joule.

On peut ainsi prévoir l'obtention de courants et d'énergies magnétiques élevés par une méthode de compression de champ magnétique dans un dispositif à inductance variable, tant que les pertes de flux restent limitées, c'est-à-dire tant que la déformation du circuit est rapide. Pour cela, nous avons envisagé l'utilisation d'un explosif gazeux beaucoup moins brisant que les explosifs solides habituellement employés. Notre choix s'est porté sur le mélange stæchiométrique d'hydrogène et d'oxygène sous pression car, pour une même densité d'énergie chimique, la pression de la vapeur d'eau produite par la détonation d'un tel mélange est au moins dix fois plus faible que celle des produits de détonation d'un explosif solide et cette pression décroît moins vite lors de l'accélération des parois mobiles; la poussée est alors plus régulière et surtout la destruction est limitée aux seules parois mobiles.

3. Propriétés du mélange stæchiométrique hydrogèneoxygène. - Dans les conditions normales de pression et de température, la combustion du mélange $2 \mathrm{H}_{2}+\mathrm{O}_{2}$ fournit 7,2 MJ.m ${ }^{-3}$ et, par conséquent, la densité d'énergie chimique sous la pression $P$ est :

$$
\frac{Q}{V}=K P \quad \text { avec } K=72 .
$$

A la pression initiale de 60 bars à laquelle nous effectuons les expériences, la densité d'énergie chimique s'élève ainsi à $432 \mathrm{MJ} \cdot \mathrm{m}^{-3}$. Cette énergie est dégagée lors de la réaction chimique. La vapeur d'eau formée est alors assimilable à un gaz parfait de coefficient isentropique de détente $\gamma=1,15$ et elle exerce une pression

$$
P_{\mathrm{e}}=(\gamma-1) \frac{Q}{V} \simeq 650 \text { bars } .
$$

Pour les produits de détonation des explosifs solides, $\gamma$ est compris entre 2,5 et 3,5 ; cela explique que, à même densité d'énergie, la pression d'explosion soit beaucoup plus élevée.

Lorsque la vapeur d'eau se détend, par exemple en déformant le récipient qui la contient, le travail fourni s'exprime par la variation de l'énergie interne $E_{0}-E_{\mathrm{f}}$, l'indice $O$ désignant l'état initial et l'indice $f$ l'état au moment où cesse la propulsion. On considérera comme énergie chimique de l'explosif la valeur initiale $E_{0}$ L'approximation d'une détente adiabatique et homogène est pratiquement toujours justifiée ; alors, d'après la loi $P V^{\gamma}=$ Cte, le travail fourni par la détente $W$ vaut :

$$
W=E_{0}\left[1-\left(\frac{V_{0}}{V_{\mathrm{f}}}\right)^{\gamma-1}\right] .
$$

Sur la figure 2 nous avons tracé le rendement $\eta=W / E_{0}$ en fonction du rapport de détente $V / V_{0}$. Nous remarquons que $\eta$ est nettement plus petit que 1 pour toutes les valeurs de $V / V_{0}$ que l'on sait réaliser simplement, et que l'accroissement de $\eta$ avec $V / V_{0}$ est sensible, même pour des valeurs élevées de $V / V_{0}$. Il en résulte qu'il faut manifestement rechercher les systèmes qui assurent le plus grand rapport $V / V_{0}$ possible afin d'obtenir un travail de détente élevé. 


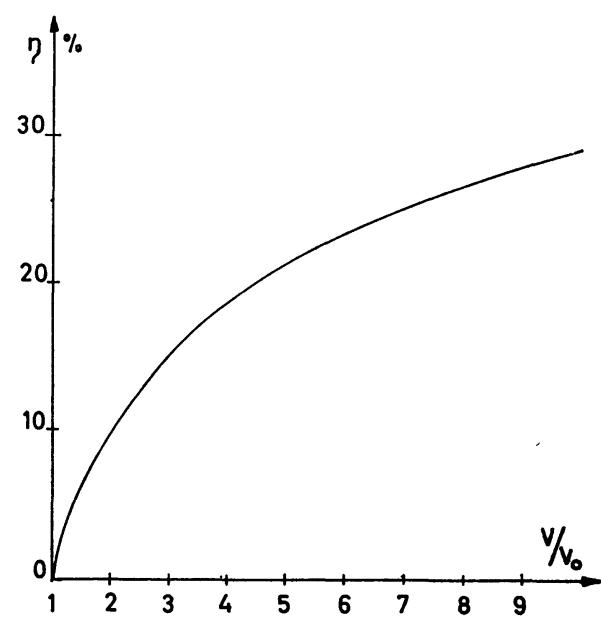

Fig. 2. - Variation du travail de détente de la vapeur d'eau rapporté à l'énergie chimique en fonction du taux de détente $V / V_{0}$.

4. Choix d'une structure de réalisation et de ses dimensions. - L'utilisation d'un explosif gazeux impose la tenue de la pression initiale de façon étanche. Le moyen le plus simple consiste à contenir le mélange gazeux dans un tube cylindrique fermé aux extrémités. La structure coaxiale du générateur s'impose dès lors car elle est simple de réalisation et mécaniquement très résistante; nous l'avons adoptée.

L'inductance variable du circuit électrique est celle du système coaxial représenté schématiquement sur la figure 3. La paroi mobile est un tube métallique fin (1)

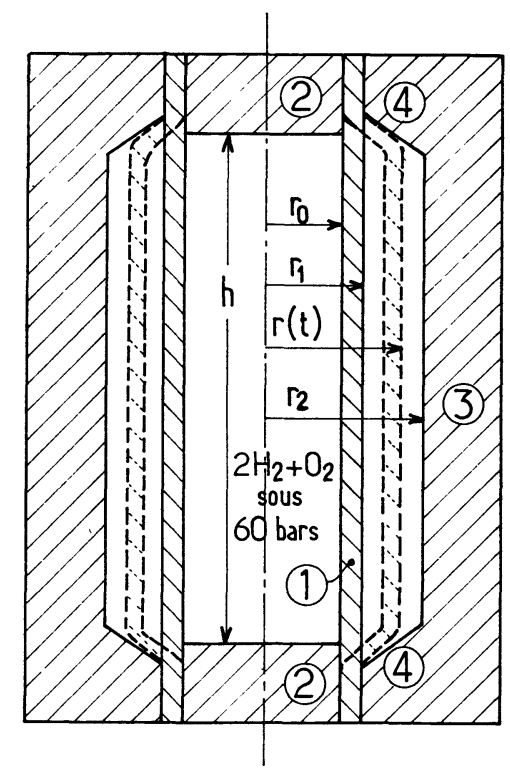

FIG. 3. - Schéma du générateur Cylex.

placé coaxialement à l'intérieur d'une carcasse en acier (3). Le mélange gazeux $2 \mathrm{H}_{2}+\mathrm{O}_{2}$ sous pression est contenu dans ce tube qui est fermé, de façon étanche, par deux bouchons appropriés (2). La carcasse se raccorde au tube mobile par deux parties coniques massives (4). La détonation de l'explosif gazeux, amorcée suivant l'axe de révolution du système par une explosion de fil, exerce une pression importante sur la paroi interne du tube qui est ainsi déformé plastiquement et accéléré. Si l'on crée un flux magnétique initial entre le tube et la carcasse, l'expansion radiale rapide du tube comprime le champ magnétique jusqu'à ce que la pression magnétique croissante l'arrête (si les pertes de flux ne sont pas trop élevées). L'énergie cinétique acquise sous la poussée de la vapeur d'eau est ainsi transformée partiellement en énergie magnétique. Cette dernière peut être transférée à une charge à l'aide d'un commutateur utilisant le phénomène d'explosion de fil ou de feuille [11].

Les performances que peut fournir un tel dispositif dépendent de la qualité des conversions successives d'énergie chimique-cinétique puis cinétique-magnétique. Cette distinction peut se faire car la pression magnétique reste faible pendant la plus grande partie du mouvement du tube et ne croît brusquement que lorsque le tube est proche de la carcasse. L'étude de ces transformations d'énergie permet de définir les dimensions d'un générateur suivant l'énergie finale recherchée.

5. Transformation de l'énergie chimique en énergie cinétique. - La masse de l'explosif étant très petite devant la masse du tube, il est légitime de négliger l'énergie cinétique de la vapeur d'eau devant celle du tube. Par conséquent, le travail fourni par la détente de la vapeur d'eau se retrouve presque intégralement en énergie cinétique du tube et en travail de déformation qui, comme nous le verrons, devient relativement important. De toutes façons, pour obtenir la plus grande énergie cinétique pour un tube donné, il faut emmagasiner l'énergie chimique la plus grande possible et obtenir le plus grand rendement possible. Ainsi la pression de l'explosif gazeux doit être la pression maximum admissible sans une déformation excessive du tube qui nuierait au bon fonctionnement du générateur. Ensuite on doit rechercher la plus grande détente possible de la vapeur d'eau sans rupture de la paroi du tube. Ces considérations nous ont fait adopter des tubes en aluminium A5 recuit, matériau léger, bon conducteur et caractérisé par un grand allongement à la rupture.

Des expériences préliminaires de déformation plastique de tubes en aluminium de $80 \mathrm{~mm}$ de diamètre intérieur et de $6 \mathrm{~mm}$ d'épaisseur dans des conditions statiques (sous une pression hydraulique) et dynamiques (sous la poussée de l'explosion du mélange $2 \mathrm{H}_{2}+\mathrm{O}_{2}$ ) ont déterminé :

- la pression interne maximum admissible,

- l'allongement à la rupture lors d'une déformation plastique rapide,

- le mouvement d'expansion radiale du tube,

- le travail absorbé par la déformation.

5.1 DÉTERMINATION DE LA PRESSION INTERNE MAXIMUM. - La courbe de traction rationnelle $\sigma=k \varepsilon^{n}$ (où l'allongement est défini par $\varepsilon=\log l / l_{0}$, 
$\left.k=14,4 \mathrm{~kg} \cdot \mathrm{mm}^{-2}, n=0,278\right) \mathrm{du}$ matériau utilisé, communiquée par le centre de recherches de Voreppe G. P., permet théoriquement de déterminer la pression maximum admissible. Cette courbe ne fait cependant pas apparaître de limite élastique et ne présente pas de coude franc ; ainsi le choix d'une contrainte limite est malaisé, on peut la situer au voisinage de $5 \mathrm{kgf} . \mathrm{mm}^{-2}$. Nous avons donc effectué des essais de déformation quasi statique de ces tubes en les remplissant d'huile sous pression.

Pour différentes pressions appliquées par ordre croissant, nous avons mesuré le diamètre extérieur du tube en différentes sections droites. Les résultats de ces mesures montrent qu'au-delà de 60 bars la déformation augmente rapidement. Le tube qui est resté symétrique jusque-là, se cintre ensuite et prend une flèche de plus en plus importante, qui dépasse $10 \mathrm{~mm}$ à 70 bars pour une longueur de $700 \mathrm{~mm}$.

Nous avons ainsi fixé la pression maximum admissible dans ces tubes à 60 bars. La contrainte tangentielle et la déformation s'élèvent alors respectivement à $4,5 \mathrm{kgf} . \mathrm{mm}^{-2}$ et $2 \%$.

5.2 RUPTURE DU TUBE LORS DE LA DÉFORMATION PLASTIQUE RAPIDE. - Dans une carcasse permettant un taux de dilatation en rayon du tube voisin de 2, nous avons fait exploser une série de tubes. Les déformations finales obtenues sont d'autant plus importantes et symétriques que la pression initiale de l'explosif gazeux est élevée. Pour une pression de 10 à 20 bars, la forme finale du tube après explosion est très dissymétrique, la rupture de la paroi apparaît suivant une seule génératrice (cf. Fig. 4). L'aspect est semblable à celui obtenu après une déformation quasi statique. Avec une pression de 60 bars, la symétrie cylindrique initiale est beaucoup mieux conservée. De nombreuses fractures apparaissent simultanément suivant les génératrices, elles sont réparties assez régulièrement tout autour du tube (cf. Fig. 4).
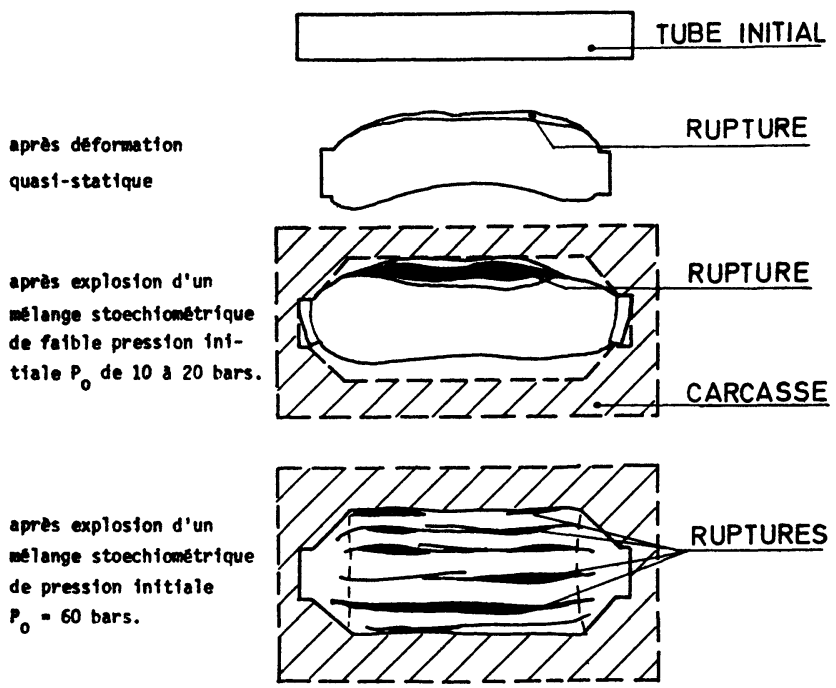

Fig. 4. - Aspects des tubes en A.5 recuits utilisés dans l'expérience Cylex.
Pour déterminer la déformation du tube lorsque débutent les premières fractures, nous avons garni intérieurement la carcasse pour obtenir des portions cylindriques de différents diamètres : 130, 140, 150 et $160 \mathrm{~mm}$ (rappelons que le tube a un diamètre intérieur de $80 \mathrm{~mm}$ et une épaisseur de $6 \mathrm{~mm}$ ).

L'examen des tubes après explosion montre que les premières strictions de la paroi ont lieu au voisinage du diamètre de $140 \mathrm{~mm}$ et que, pour $150 \mathrm{~mm}$, les ruptures présentent des bords écartés de quelques millimètres. Elles apparaissent donc pour un diamètre voisin de $145 \mathrm{~mm}$. Nous n'avons pas observé de corrélations entre les défauts initiaux de l'épaisseur du tube et ces strictions. Celles-ci débutent à partir de grains du métal dont la taille est de plusieurs millimètres; elles ont lieu parfois sur la surface interne du tube, parfois sur la surface externe et conduisent à la rupture. L'allongement à la rupture, déterminé par la variation relative de l'épaisseur du tube, est dans ces conditions :

$$
\frac{e_{0}}{e_{\mathrm{f}}}=1,65
$$

où $e_{\mathrm{f}}$ et $e_{0}$ sont respectivement les épaisseurs finales et initiales du tube.

Par la suite le taux de dilatation maximum des tubes a été fixé à 1,6 pour éviter la rupture de la paroi. Dans ces conditions la détente en volume de la vapeur d'eau issue de l'explosion est de 2,7 et le travail fourni par la détente atteint $13 \%$ de l'énergie chimique. Ce travail fourni est transformé en partie en énergie cinétique du tube.

5.3 MOUVEMENT D'EXPANSION RADIALE DU TUBE. La mesure de la position de la paroi extérieure du tube au cours de son expansion est effectuée à l'aide de sondes de contact électrique. Chaque sonde de contact est constituée par un fil fin en tungstène qui, lorsqu'il touche la paroi du tube, permet de décharger une capacité $C$ à travers une résistance $R$. Les sondes sont de longueur différente. Au cours de son mouvement radial, le tube rencontre successivement les sondes. Le premier contact établit la décharge de la capacité $\mathrm{C}$, les suivants modifient les paramètres de la décharge. La mesure du courant de la décharge et de sa dérivée indiquent avec précision les instants des différents contacts.

Sur la figure 5, nous avons porté, pour un essai typique, les instants de contact en fonction de la position des sondes. Les points obtenus se situent sur une parabole puis sur une tangente à cette parabole. La vitesse du tube vaut ainsi, avec une précision de $10 \%$, $v(t)=1,9 \times 10^{6} t \mathrm{~ms}^{-1}$ jusqu'à $r(t)=63 \times 10^{-3} \mathrm{~m}$ puis $v(t)=250 \mathrm{~ms}^{-1}$ jusqu'à $r(t)=70 \times 10^{-3} \mathrm{~m}$.

5.4 ENERGIE ABSORBÉE PAR LA DÉFORMATION DU TUBE. - L'énergie $W_{\mathrm{d}}$ absorbée par la déformation du tube est la différence entre le travail $W=\eta E_{0}$ fourni par la détente de la vapeur d'eau issue de 


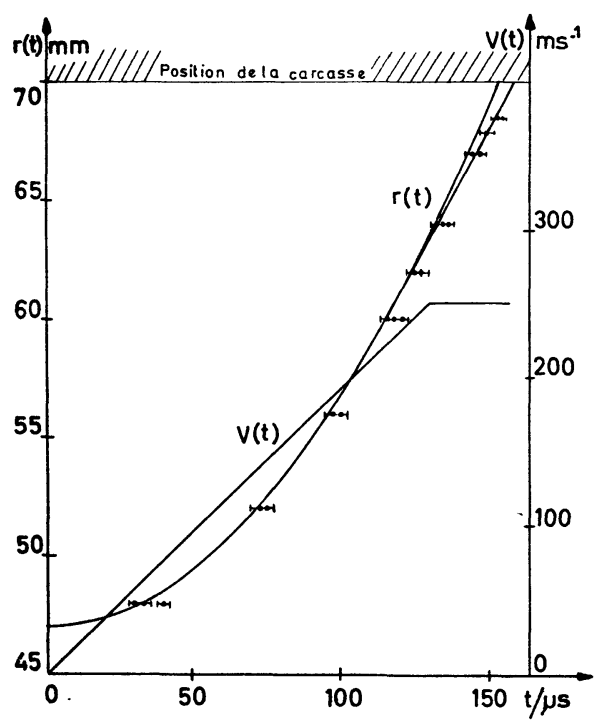

FIG. 5. - Mouvement du tube.

l'explosion du mélange $2 \mathrm{H}_{2}+\mathrm{O}_{2}$ et l'énergie cinétique $W_{\mathrm{c}}$ acquise par le tube.

Le travail fourni par la détente se calcule à partir de la relation (6). Dans notre montage, le volume intérieur du tube est $2,2 \times 10^{-3} \mathrm{~m}^{3}$, la densité d'énergie chimique étant de $430 \mathrm{MJ} . \mathrm{m}^{-3}$,

$$
E_{0}=950 \mathrm{~kJ} \text {. }
$$

En considérant la déformation finale du tube avec $\gamma=1,15$ nous obtenons :

$$
W=\eta E_{0}=127 \mathrm{~kJ} .
$$

Calculons l'énergie cinétique acquise par le tube. La conservation de la section du tube au cours de la déformation plastique entraîne qu'un point situé à l'intérieur de la paroi du tube, à un rayon $u$, est animé d'une vitesse $V_{u}$ supérieure à la vitesse $V$ de la surface extérieure :

$$
V_{u}=\frac{r(t) \cdot V(t)}{u}
$$

et par conséquent l'énergie cinétique du tube de masse $m$ s'exprime par :

$$
W_{\mathrm{c}}=m V^{2}(t) \frac{r^{2}(t)}{r_{1}^{2}-r_{0}^{2}} \log \frac{r(t)}{r_{0}} .
$$

Pour

$$
r(t)=70 \times 10^{-3} \mathrm{~m}, \quad W_{\mathrm{c}}=62 \mathrm{~kJ} .
$$

Finalement, le travail nécessaire pour déformer le tube, dans nos conditions d'expérience, s'élève à

$$
W_{\mathrm{d}}=65 \mathrm{~kJ}
$$

c'est-à-dire à environ l'énergie cinétique acquise par le tube.

Le travail de déformation calculé à partir de la REVUE DE PHYSIQUE APPLIQUÉE. - T. 11, N॰ 3, MAI 1976 courbe de traction rationnelle et correspondant à une déformation lente

$$
\sigma=k \varepsilon^{n} \quad \text { avec } k=14,4 \times 10^{7}, \quad n=0,278
$$

conduit à

$$
W_{\mathrm{d}}=V_{l} \frac{k}{n+1} \varepsilon^{n+1}=29 \mathrm{~kJ} .
$$

Lors de nos essais en déformation rapide le travail de déformation atteint plus de deux fois cette valeur.

L'étude de la transformation énergie chimiquecinétique, la pression initiale de l'explosif gazeux étant fixée par l'épaisseur de la paroi du tube, ne permet pas de guider le choix de la taille d'un générateur. D'autres considérations, provenant de l'étude de la transformation énergie cinétique-magnétique, détermineront les dimensions radiales.

6. Transformation de l'énergie cinétique en énergie magnétique. - Lors du freinage de la paroi par la pression magnétique, l'énergie cinétique acquise est transformée en énergie électrique. La partie utile de celle-ci correspond au champ magnétique qui se trouve dans l'air. C'est elle qui est récupérable lors d'une commutation rapide, le champ magnétique qui se retrouve dans le métal étant pratiquement perdu, puisqu'il reste gelé à l'intérieur des conducteurs au-delà de l'épaisseur de peau. Par ailleurs, le courant produit échauffe les parois du générateur par effet Joule. Ainsi une partie de l'énergie cinétique du tube est convertie en énergie irrécupérable dans les conducteurs. Ces pertes, liées au phénomène de diffusion du champ magnétique, ont été traitées en détail $[8,12]$. L'étude effectuée a montré en particulier que le montage à courant axial est plus intéressant au point de vue des performances que celui à courant azimutal. Comme par ailleurs sa réalisation est plus simple, nous ne considérerons désormais que la première structure.

Ici, nous donnerons seulement une description utilisant un simple circuit composé d'une inductance $L(t)$ et d'une résistance $R(t)$ variables en série qui permet de prévoir de façon assez correcte le fonctionnement des générateurs et de dégager l'influence des principaux paramètres.

6.1 Etude De L'équation Électrique AVEC L'APPROXIMATION DU COURANT EXPONENTIEL. - Nous allons résoudre l'éq. (1) :

$$
\frac{\mathrm{d}}{\mathrm{d} t}(L I)+R I=0
$$

et par conséquent calculer $L(t)$ et $R(t)$ en admettant une croissance exponentielle du courant dans le générateur :

$$
I(t)=I_{0} \mathrm{e}^{t / \tau}
$$

Cette approximation est justifiée par le fait suivant : la croissance du courant dans les générateurs étudiés a une allure exponentielle, sauf au tout début de la compression où la croissance du courant est plus lente 
mais alors le tube est loin de la carcasse et les pertes de flux sont peu importantes.

Dans ces conditions, l'expression de la résistance est celle, classique, que l'on obtient lorsque l'on considère le courant réparti uniformément dans l'épaisseur de peau $\delta$ donnée par

$$
\delta=\sqrt{\frac{\tau}{\mu_{0} \sigma}} .
$$

L'éq. (1) prend la forme :

$$
\frac{\mathrm{d}}{\mathrm{d} t} L\{r(t)\}+\frac{L\{r(t)\}}{\tau}+R\{r(t)\}=0
$$

dans laquelle le courant $I(t)$ ne figure plus. Elle lie la position et la vitesse du liner à la constante de temps $\tau$. Pour une structure coaxiale avec un courant axial avec les valeurs

$$
\left.\begin{array}{l}
L\{r(t)\}=\frac{\mu_{0} h}{2 \pi} \log \frac{r_{2}}{r(t)} \\
R\{r(t)\}=\left(\frac{\mu_{0}}{\sigma_{l} \tau}\right)^{1 / 2} \frac{h}{2 \pi}\left[\frac{1}{r(t)}+\left(\frac{\sigma_{l}}{\sigma_{\mathrm{c}}}\right)^{1 / 2} \frac{1}{r_{2}}\right]
\end{array}\right\}
$$

(où $\sigma_{l}$ et $\sigma_{\mathrm{c}}$ sont les conductivités du tube et de la carcasse) elle conduit à une relation valable à chaque instant :

$$
\begin{aligned}
v\{r(t)\}=\frac{1}{\left(\mu_{0} \sigma_{l} \tau\right)^{1 / 2}}\left[1+\frac{r(t)}{r_{2}}\left(\frac{\sigma_{l}}{\sigma_{\mathrm{c}}}\right)^{1 / 2}\right]+ \\
+\frac{r(t)}{\tau} \log \frac{r_{2}}{r(t)} .
\end{aligned}
$$

Dans toute la suite des calculs nous considérons que les conductivités $\sigma_{l}$ et $\sigma_{\mathrm{c}}$ sont indépendantes du temps, ce qui revient à admettre que les pertes par effet Joule (liées aux pertes de flux) ne modifient pas les conductivités. Cette hypothèse est confirmée par les résultats expérimentaux.

Ces relations vont nous permettre de calculer le coefficient de conservation du flux

$$
\lambda=\exp -\int_{0}^{t} \frac{R}{L} \mathrm{~d} t
$$

et celui de la multiplication de l'énergie

$$
\frac{W}{W_{0}}=\Gamma \lambda^{2} \quad\left(\Gamma=L / L_{0}\right)
$$

Compte tenu des relations (7) et (8), nous obtenons :

$$
\begin{aligned}
& \log \lambda(r)=\frac{1}{\tau} \int_{r_{1}}^{r(t)} \times \\
& \times \frac{\frac{1}{\left(\mu_{0} \sigma_{l} \tau\right)^{1 / 2}}\left[1+\frac{r(t)}{r_{2}}\left(\frac{\sigma_{l}}{\sigma_{c}}\right)^{1 / 2}\right]+\frac{r(t)}{\tau} \log \frac{r_{2}}{r(t)}}{-\int_{r_{1}}^{r(t)} \frac{\mathrm{d} r}{r(t) \log \frac{r_{2}}{r(t)}}}
\end{aligned}
$$

où $r_{1}=r(0)$ est le rayon extérieur initial du tube et $r_{2}$ le rayon interne de la carcasse.

Le calcul de la dernière intégrale est immédiat, il donne :

$$
\log \frac{\log \frac{r_{2}}{r(t)}}{\log \frac{r_{2}}{r_{1}}} .
$$

La première, non intégrable par des fonctions usuelles, peut l'être simplement en utilisant un algorithme de la forme :

$$
r \log r=a r^{2}+b r+c
$$

où $a, b, c$ sont des constantes que l'on calcule de manière que

$$
\frac{\left|a r^{2}+b r+c-r \log r\right|}{r \log r} \leqslant \varepsilon
$$

pour l'intervalle de variation de $r: r_{1} \leqslant r \leqslant r_{2}$. On peut prendre $\varepsilon$ aussi petit que l'on veut, son choix définit la précision du calcul. Mais, bien évidemment la lourdeur du calcul est liée à ce choix.

Nous obtenons en définitive pour $\lambda$ l'expression :

$$
\begin{aligned}
\log \lambda & =\log \frac{\log \frac{r_{2}}{r(t)}}{\log \frac{r_{2}}{r_{1}}}+ \\
+ & \frac{1}{N}\left[\log \frac{2 \operatorname{ar}(t)+M-N}{2 \operatorname{ar}(t)+M+N}-\log \frac{2 a r_{1}+M-N}{2 a r_{1}+M+N}\right]
\end{aligned}
$$

où nous avons posé :

$$
\left.\begin{array}{c}
M=b-\frac{1}{r_{2}}\left(\frac{\tau}{\mu_{0} \sigma_{\mathrm{c}}}\right)^{1 / 2}-\log r_{2} \\
N=\left[\left[b-\frac{1}{r_{2}}\left(\frac{\tau}{\mu_{0} \sigma_{\mathrm{c}}}\right)^{1 / 2}-\log r_{2}\right]^{2}-\right. \\
\left.-4 a\left[c-\left(\frac{\tau}{\mu_{0} \sigma_{l}}\right)^{1 / 2}\right]\right]^{1 / 2}
\end{array}\right\}
$$

La multiplication d'énergie magnétique $\Gamma \lambda^{2}$ se calcule aisément à partir de (3), (10) et (11).

Ces expressions littérales permettent d'étudier graphiquement les variations de $\lambda(r)$ et de $\Gamma \lambda^{2}(r)$ suivant les valeurs de $r_{2}$ et de $r_{2} / r_{1}$.

6.2 ETUDE DE L'INFLUENCE DE LA DIMENSION RADIALE SUR LA CONSERVATION DU FLUX - Nous considérons une suite de montages homothétiques se déduisant les uns des autres en doublant les dimensions radiales du montage précédent et nous calculons dans chaque cas le coefficient de conservation du flux $\lambda(r)$ en fonction de la position du tube. Pour tous ces montages, nous fixons le rapport $r_{2} / r_{1}$ à la valeur du taux de dilatation maximum de nos expériences soit $r_{2} / r_{1}=1,53$.

Sur la figure 6, nous avons tracé les variations de

$$
\lambda\left(\frac{r}{r_{1}}\right) \text { pour } 1 \leqslant \frac{r}{r_{1}} \leqslant \frac{r_{2}}{r_{1}}=1,53
$$


pour les différentes valeurs de $r_{2}$ marquées sur la figure. Ces courbes montrent que si $r_{2}$ est grand, de

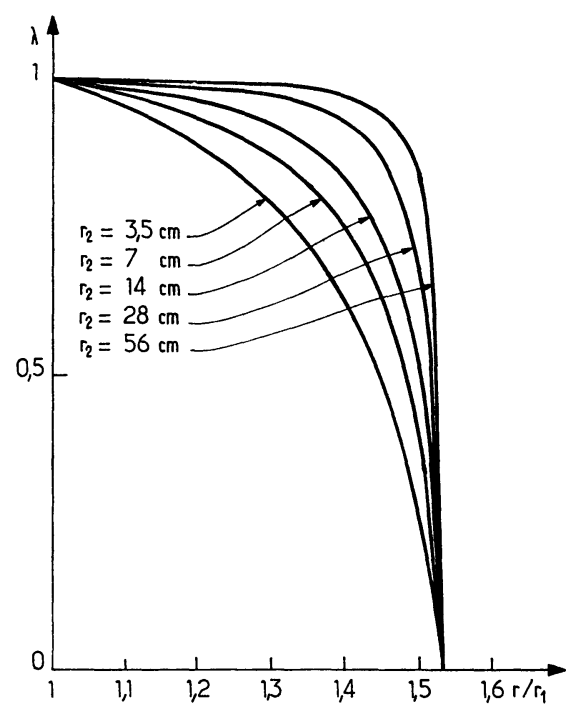

Fig. 6. - Variation du coefficient de conservation du flux en fonction de la position du liner, pour différents rayons $r_{2}$ de la carcasse, avec $r_{2} / r_{1}=1,53$.

l'ordre de $0,5 \mathrm{~m}$, le flux est pratiquement conservé jusqu'à ce que le tube soit très proche de la paroi, la multiplication d'énergie magnétique correspondante peut être très importante dans ce cas. Elle n'est plus limitée par les pertes de flux. En pratique, elle serait limitée par les défauts de cylindricité et de centrage du tube. Lorsque $r_{2}$ est petit, de l'ordre de quelques centimètres, les pertes de flux sont importantes dès le début $\mathrm{du}$ mouvement du tube, par conséquent ce montage ne permet pas une bonne transformation de l'énergie cinétique en énergie magnétique.

Sur la figure 7 nous avons porté la variation de la valeur maximum de $\Gamma \lambda^{2}$ en fonction du rayon $r_{2}$ de la

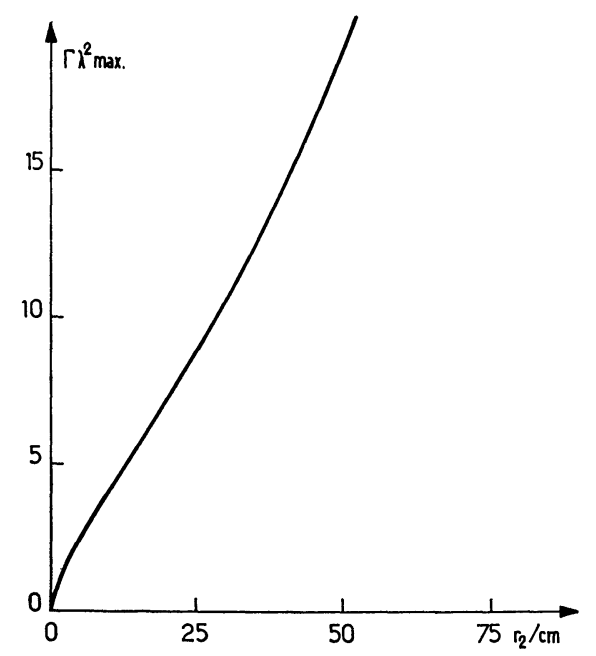

Fig. 7. - Valeur maximum de la multiplication de l'énergie magnétique en fonction du rayon $r_{2}$ de la carcasse pour $r_{2} / r_{1}=1,53$. carcasse, dans les mêmes conditions que précédemment. Cette courbe est presque une droite, elle montre que le maximum de multiplication d'énergie magnétique est pratiquement proportionnel à la dimension radiale du montage. Il y a donc intérêt à choisir les dimensions les plus grandes possible pour un rapport $r_{2} / r_{1}$ donné.

7. Choix des dimensions du montage. - L'étude que nous venons d'effectuer de la transformation d'énergie chimique en énergie cinétique et de cette dernière en énergie magnétique permet de définir, avec une bonne précision, les dimensions minimales d'un montage d'essai afin qu'il soit léger et relativement aisé à manipuler, et qu'il fournisse cependant des performances (multiplication de l'énergie magnétique $\sim 3$, coefficient de conservation du flux $\sim 0,5$ ) permettant de conclure sur la possibilité de réaliser un générateur à compression de champ magnétique à explosif gazeux à un niveau d'énergie bien supérieur (10 ou $20 \mathrm{MJ}$ par exemple).

Ce compromis nous conduit à choisir un rayon intérieur de la carcasse $r_{2}=7 \mathrm{~cm}$, un rayon extérieur du tube $r_{1}=4,6 \mathrm{~cm}$ (déterminé par $r_{2} / r_{1}=1,53$ ) et une longueur du tube $h_{0}=43 \mathrm{~cm}$.

Les dimensions du montage étant ainsi fixées, le bilan des énergies permet de déterminer l'énergie magnétique initiale correspondant à $\lambda \simeq 0,5$ et $\Gamma \lambda^{2}=3$. L'énergie chimique atteint, pour une pression de 60 bars du mélange gazeux explosif

$$
E_{0} \simeq 1 \mathrm{MJ} .
$$

Dans ces conditions, comme nous l'avons vu précédemment, l'énergie cinétique acquise par le tube est :

$$
W_{\mathrm{c}} \simeq 65 \mathrm{~kJ} \text {. }
$$

La relation (8) permet d'évaluer la vitesse du tube au moment du maximum de l'énergie magnétique. On obtient $v \simeq 100 \mathrm{~m} / \mathrm{s}$, ce qui correspond à une énergie cinétique de $10 \mathrm{~kJ}$. La variation d'énergie cinétique est donc

$$
\Delta W_{\mathrm{c}} \simeq 55 \mathrm{~kJ} .
$$

L'énergie magnétique utile finale $W$ est égale à l'énergie perdue par diffusion puisque $\lambda \simeq 0,5$, elle est d'autre part égale à trois fois l'énergie magnétique initiale $W_{0}$ puisque $\Gamma \lambda^{2}=3$. D'où :

$$
\begin{aligned}
\Delta W_{\mathrm{c}}+W_{0} & =6 W_{0} \\
W_{0} & \simeq 10 \mathrm{~kJ} .
\end{aligned}
$$

L'énergie magnétique maximum est par conséquent

$$
W_{\mathrm{m}}=30 \mathrm{~kJ} \text {. }
$$

8. Le générateur à compression de champ magnétique. - Le générateur a déjà été décrit schématiquement ci-dessus. Nous allons maintenant préciser certains points et pour cela nous reporter à la figure 8 . 


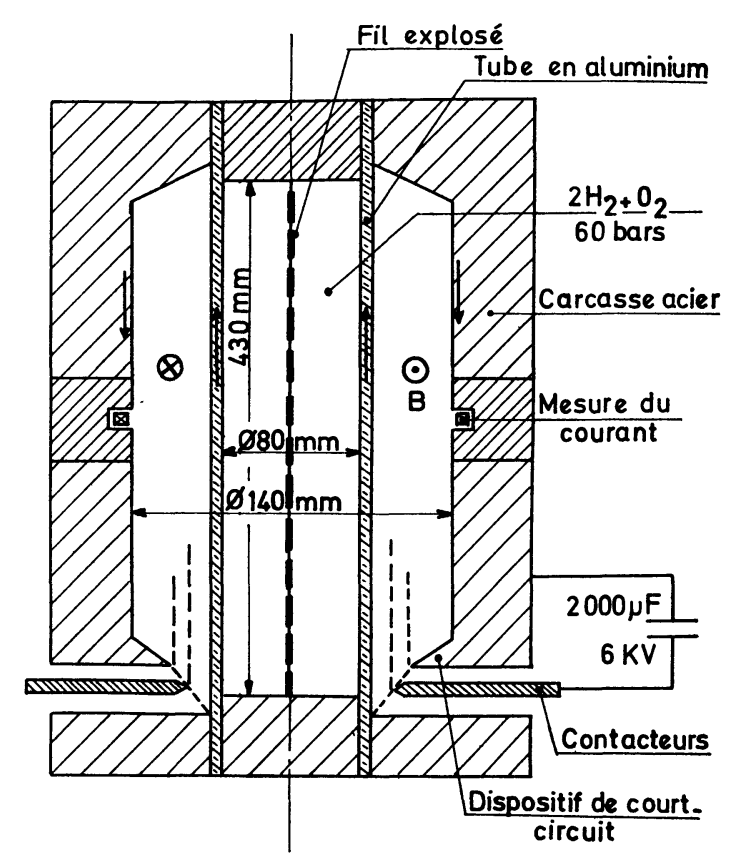

Fig. 8. - Schéma du générateur Cylex.

Le tube est en aluminium A5 recuit, les deux bouchons en acier inoxydable. Ces derniers assurent, outre l'étanchéité du volume intérieur au tube, le centrage mécanique de celui-ci par rapport à la carcasse.

La carcasse a un double rôle :- un rôle électrique; elle forme le conducteur extérieur coaxial au liner et doit assurer le passage du courant axial, - un rôle mécanique; elle doit pouvoir encaisser le choc du liner en bout de course sans être détériorée. Pour assurer ces deux fonctions la carcasse est constituée de deux cylindres concentriques. Le cylindre intérieur est relativement mince, il est réalisé en bronze bon conducteur. Le cylindre extérieur, en acier, est épais, il forme le blindage mécanique du dispositif. L'expérience nous a montré qu'il y avait une bonne transmission des efforts entre les deux cylindres car le cylindre intérieur n'a jamais été détérioré au cours des essais. Aux deux extrémités, la surface intérieure de la carcasse se raccorde au tube par une partie conique. Afin de pouvoir démonter cet ensemble et changer le tube déformé après un tir, la carcasse est constituée de trois tronçons cylindriques. La partie centrale, la plus légère, comporte les dispositifs de mesure.

La production du mélange stæchiométrique hydrogène-oxygène est réalisée par électrolyse sous pression d'une solution de potasse. Ce procédé, qui évite tout stockage, est d'un emploi sûr et simple.

La source annexe d'énergie électrique qui doit créer l'énergie magnétique initiale dans le système coaxial tube-carcasse, d'une inductance de $40 \mathrm{nH}$ environ, est un banc de 4 condensateurs Haefely de faible inductance interne groupés en parallèle. Les caractéristiques globales sont les suivantes :

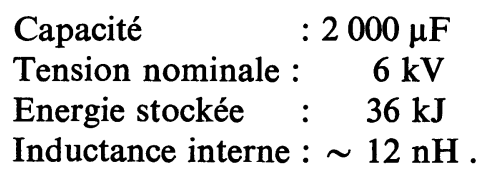

La liaison entre le banc de condensateurs et le générateur à compression de champ est réalisée à l'aide de larges plaques de cuivre (largeur : $80 \mathrm{~cm}$, épaisseur : $2 \mathrm{~mm}$ ), séparées par une feuille d'isolant d'épaisseur $1 \mathrm{~mm}$; elles sont maintenues serrées l'une contre l'autre à l'aide de renforts en tôle d'acier d'épaisseur $5 \mathrm{~mm}$. Le banc de condensateurs est placé à proximité du montage et la longueur de la liaison est environ la largeur des plaques. L'inductance de cette liaison est ainsi de quelques $\mathrm{nH}$ au plus.

Pour créer l'énergie magnétique initiale dans le générateur on doit décharger le banc de condensateurs dans le système coaxial tube-carcasse, puis on doit courtcircuiter les bornes d'alimentation du générateur pour découpler son fonctionnement de celui du banc. La décharge doit être synchronisée avec la mise en mouvement du tube, et le court-circuit doit s'effectuer lors du maximum du courant de la décharge afin de disposer du maximum d'énergie magnétique initiale. Aussi avonsnous utilisé le tube lui-même pour effectuer ces fermetures de circuits : au début de son mouvement d'expansion radiale, il entre en contact avec 12 électrodes disposées radialement à une même distance $r_{\mathrm{d}}$ de l'axe de révolution du générateur, et établit ainsi la décharge du banc de condensateurs.

Ensuite, poursuivant son mouvement, il entre en contact au rayon $r_{\mathrm{cc}}$ avec l'extrémité conique de la carcasse, et établit le court-circuit. Le champ magnétique initial est alors piégé entre le liner et la carcasse et sa compression va s'effectuer indépendamment du circuit d'alimentation du générateur. La synchronisation de l'instant de court-circuit avec le maximum d'intensité de la décharge s'obtient par le réglage préalable de la position des électrodes de contact.

Pour amorcer la détonation du mélange gazeux $2 \mathrm{H}_{2}+\mathrm{O}_{2}$, nous utilisons l'explosion violente d'un fil de cuivre placé suivant l'axe du tube et dans lequel on décharge rapidement un banc de condensateurs. Comme le mélange est à la pression initiale de 60 bars, il faudrait mettre en jeu par centimètre de longueur de fil une énergie de $80 \mathrm{~J}$ et une tension de 2,5 à $3 \mathrm{kV}$ soit au moins $100 \mathrm{kV}$ dans notre cas. Pour éviter ces tensions élevées qui posent de sérieux problèmes d'isolement, nous avons utilisé un fil formé de tronçons de sections fortes et faibles alternant régulièrement (Fig. 9). Lors de la décharge du banc de condensateurs, seules les parties à faible section absorbent de l'énergie, sont vaporisées et amorcent la détonation. Dans notre

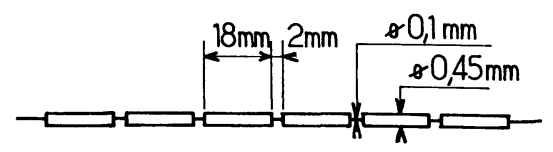

FIG. 9. - Fil d'amorçage de la détonation. 
montage où les tronçons de faible section, de longueur $2 \mathrm{~mm}$, sont distants de $18 \mathrm{~mm}$ c'est-à-dire de près de la moitié du rayon intérieur du tube $\left(r_{0}=40 \mathrm{~mm}\right)$, l'expérience a montré que ce mode d'amorçage était satisfaisant car l'expansion du tube était bien cylindrique Le fil est fabriqué en disposant électrolytiquement du cuivre sur les parties non masquées d'un fil de cuivre de $0,1 \mathrm{~mm}$ de diamètre ; son emploi permet de diviser par 10 les tensions et énergies nécessaires (le banc utilisé a une énergie de $500 \mathrm{~J}$ sous $10 \mathrm{kV}$ ).

9. Essais de fonctionnement du générateur. - Nous avons déterminé, dans le cadre de l'approximation d'une croissance exponentielle du courant au cours de la compression du champ magnétique, les conditions initiales permettant la multiplication maximum de l'énergie magnétique dans le cas du montage réalisé. Nous présentons les résultats d'un essai effectué à partir de ces conditions initiales :

$\begin{array}{ll}\text { Pression initiale du mélange } & \\ \quad \text { gazeux } 2 \mathrm{H}_{2}+\mathrm{O}_{2} & : 60 \mathrm{bars} \\ \text { Energie chimique } & : 950 \mathrm{~kJ} \\ \text { Courant initial } & : 0,8 \mathrm{MA} \\ \text { Energie magnétique initiale } & : 10 \mathrm{~kJ}\end{array}$

Les calculs numériques du fonctionnement du générateur [12] ont été menés avec ces mêmes valeurs initiales. Nous avons, à fin de comparaison, tracé sur les mêmes figures les courbes expérimentales (en traits pleins) et celles calculées (en pointillés).

La courbe donnant le rayon $r(t)$ sur les figures 10 et 11 correspond à la fors aux valeurs moyennes des indications de position données par les sondes de contact et aux valeurs obtenues par le calcul numérique à partir des mesures effectuées en absence de champ magnétique. En fait les pointes des sondes placées à la même distance de l'axe n'entrent pas simultanément en contact avec le tube en mouvement. Elles mettent ainsi en évidence les défauts de symétrie (ovalisation, excen-

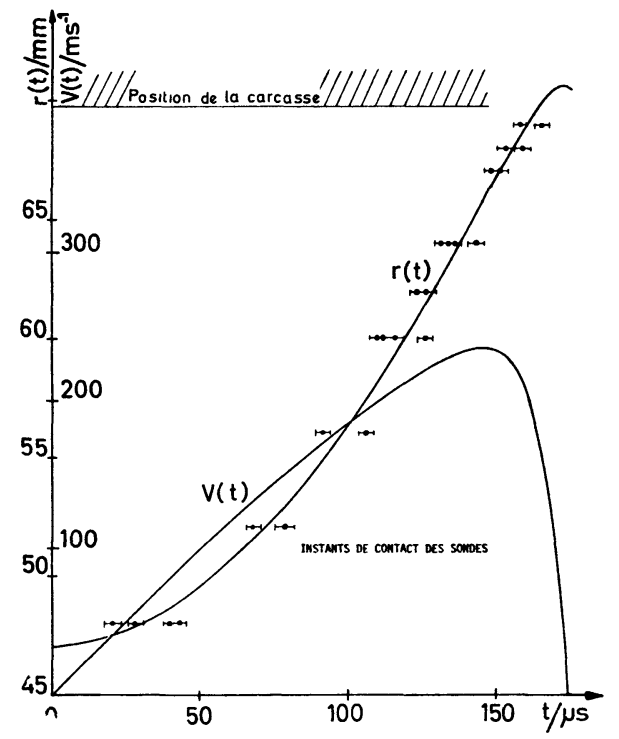

Fig. 10. - Evolution du rayon du liner $r(t)$ et de sa vitesse $V(t)$.

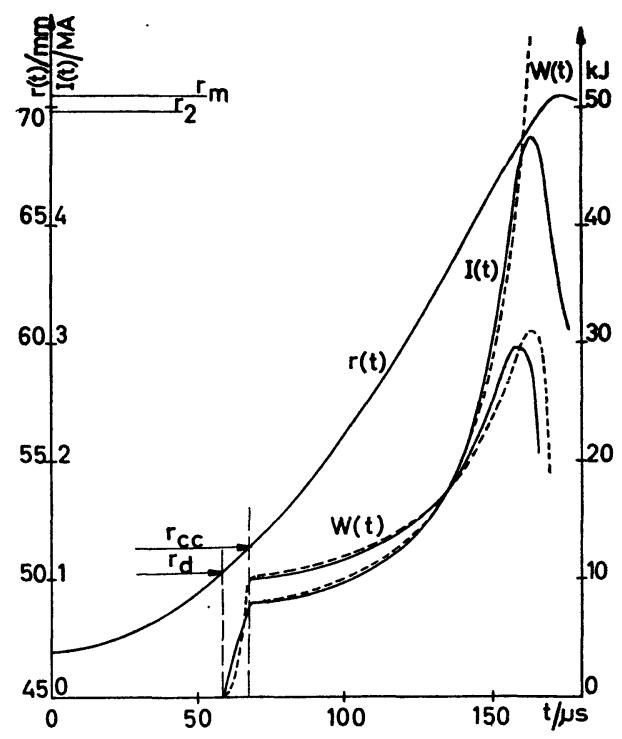

Fig. 11. - Evolution du rayon du liner $r(t)$, du courant $I(t)$, de l'énergie magnétique utile $W(t)$. Essai à $800 \mathrm{kA}$ initial :

— Courbes expérimentales; ------ Courbes théoriques.

tration) du tube. Les écarts de position par rapport au rayon moyen $r(t)$ portés sur la figure 10 peuvent atteindre le millimètre. Ces écarts, très importants sur notre montage d'essai vis-à-vis de la distance théorique d'arrêt à la carcasse, deviennent négligeables sur un véritable générateur de plus grande dimension. Ici, étant donné d'une part la feuille d'isolant enroulée à l'intérieur de la carcasse et d'autre part les gorges creusées dans la couronne centrale pour loger les sondes inductives de courant, le rayon magnétique $r_{\mathrm{m}}$ calculé de façon à donner à l'inductance du circuit sa valeur exacte ne correspond pas au rayon matériel $r_{2}$ de la carcasse plus petit : $r_{2}=69,8 \mathrm{~mm}, r_{\mathrm{m}}=70,5 \mathrm{~mm}$.

D'après le calcul, le tube, supposé parfaitement symétrique, doit s'arrêter sur le champ magnétique à

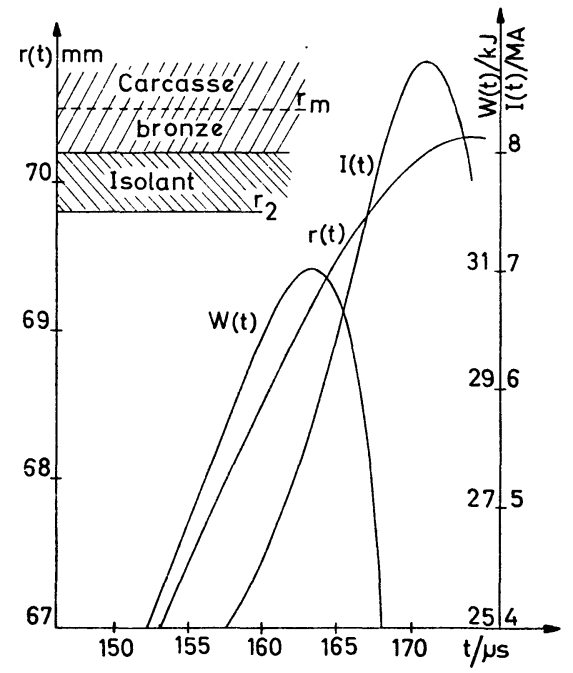

Fig. 12. - Evolutions calculées, avec un rayon de la carcasse conductrice $r_{\mathrm{m}}$, du rayon extérieur du tube $r(t)$, du courant $I(t)$, de l'énergie magnétique utile $W(t)$. Représentation détaillée lorsque $r(t)$ est voisin de $r_{\mathrm{m}}$. 
$0,2 \mathrm{~mm}$ du rayon magnétique (Fig. 12). Par conséquent, le tube doit entrer en contact avec la carcasse (vers $t=167 \mu \mathrm{s})$. En raison des défauts de symétrie du tube, le choc de ses parties les plus avancées doit même intervenir vers $160 \mu \mathrm{s}$.

La figure 11 présente les évolutions temporelles du courant $I(t)$ dans le tube et de l'énergie magnétique utile $W(t)$. Nous avons détaillé sur la figure 12 les variations calculées de $r(t), I(t), W(t)$, lorsque le tube est voisin de la carcasse. Enfin, sur la figure 13, nous présentons les variations relatives du courant $I / I_{0}$, de l'énergie magnétique $W / W_{0}$ et du flux $\lambda=\Phi / \Phi_{0}$ obtenues expérimentalement.

L'évolution du courant mesuré s'explique à partir de l'évolution du rayon moyen et des défauts de symétrie du tube. Le contact entre les pointes de décharge du banc de condensateurs et le tube correspond à la flèche marquée $r_{\mathrm{d}}$ : le courant se met à croître dans le circuit

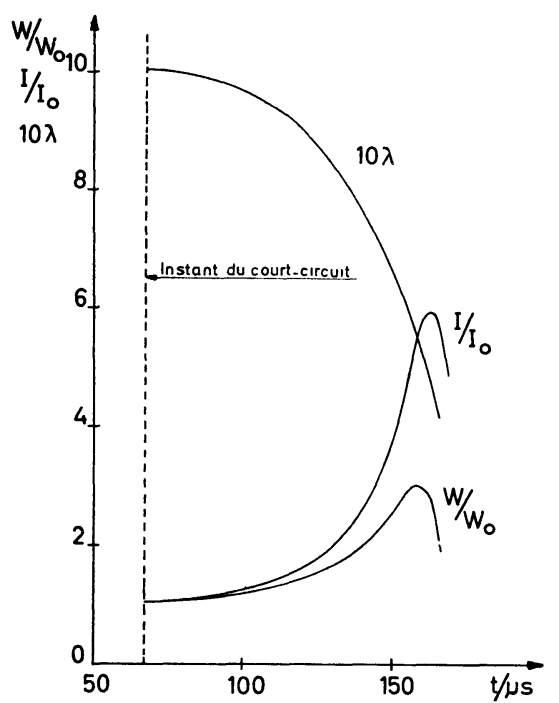

FIG. 13. - Variation relative du flux $\lambda=\Phi / \Phi_{0}$, du courant $I / I_{0}$, de l'énergie magnétique $W / W_{0}$. Essai à $800 \mathrm{kA}$ initial. coaxial. Le tube atteint ensuite la partie conique de rayon $r_{\text {cc }}$, court-circuite le banc de condensateurs et piège puis comprime le champ magnétique que dans le système coaxial. Le courant croît alors régulièrement et les calculs (courbe en traits pointillés) de diffusion du champ magnétique expliquent très bien l'évolution du courant mesuré (courbe en trait plein) jusqu'à la valeur maximum de ce dernier (4,8 MA à $t=162 \mu \mathrm{s})$.

Après cet instant les calculs indiquent que le courant doit continuer à croître très rapidement jusqu'à atteindre 8,75 MA au temps $t=171 \mu \mathrm{s}$. Expérimentalement le courant décroît. Ce désaccord s'explique très bien puisque les calculs négligent les défauts du tube et confond le rayon magnétique avec le rayon matériel intérieur de la carcasse. En réalité, dès que le tube commence à toucher la paroi de l'isolant la compression du champ magnétique s'arrête et les pertes de flux par diffusion abaissent le courant (Fig. 13).

Les mêmes observations peuvent se faire sur l'évolution de l'énergie magnétique utile tracée sur les figures 11 et 12 . Sur la figure 11, la courbe en traits pointillés correspond aux calculs théoriques, la courbe en trait plein est déduite des valeurs du rayon moyen $r(t)$ et des valeurs expérimentales du courant. Nous remarquons que, à cause de l'importance de la diffusion du champ magnétique vers la fin de la compression, l'énergie magnétique ne peut pas dépasser beaucoup le maximum obtenu expérimentalement.

Les résultats globaux de cet essai sont les suivants :

Travail fourni par la détente de la vapeur d'eau

: $127 \mathrm{~kJ}$

Vitesse maximum du tube $\quad: 235 \mathrm{~ms}^{-1}$

Energie cinétique maximum : $53 \mathrm{~kJ}$

Intensité maximum du courant : 4,8 MA

Energie magnétique maximum : $30 \mathrm{~kJ}$

Conservation du flux au maximum de l'énergie $: \sim 0,5$

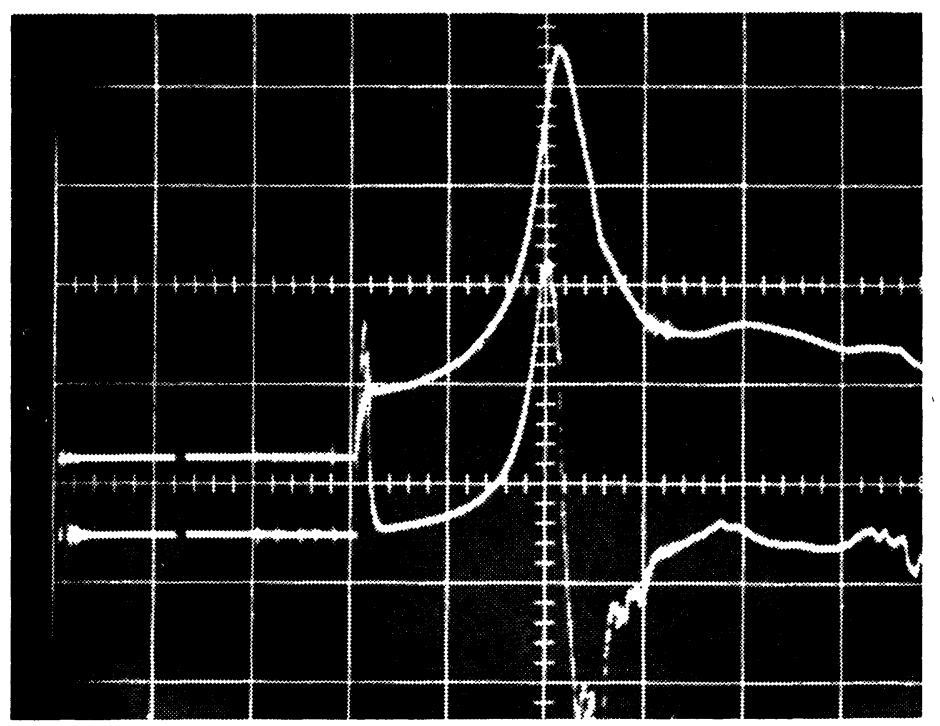

Рното 1. - Evolution du courant au cours de la compression du champ magnétique : $I: 1,15 \mathrm{MA} / \mathrm{cm}$;

$\mathrm{d} I / \mathrm{d} t: 5,5 \times 10^{10}$ A. $\mathrm{s}^{-1} / \mathrm{cm} ;$ balayage $: 50 \mu \mathrm{s} / \mathrm{cm}$. 
Ces résultats répondent aux prévisions calculées précédemment: multiplication de l'énergie magnétique par un facteur 3 avec un coefficient de conservation du flux de l'ordre de 0,5 . Les variations temporelles des différentes grandeurs : $r(t), I(t), \mathrm{W}(t), \lambda(t)$ sont en accord avec les calculs numériques.

L'enregistrement de l'évolution du courant et de sa dérivée au cours de la compression du champ magnétique est présenté sur la photo 1 .

10. Conclusion. - Les résultats expérimentaux obtenus prouvent la possibilité de réaliser un générateur à compression de champ magnétique utilisant un explosif gazeux et dont le fonctionnement n'entraîne aucune destruction autre que celle du tube déformé.
Nous avons déterminé l'énergie absorbée par la déformation plastique rapide du tube, (elle est plus du double de celle calculée en statique), ce qui nous a permis de décrire, avec un excellent accord théorie-expérience, le fonctionnement du générateur.

Avec des dimensions radiales supérieures, les pertes de flux diminuent, la distance d'arrêt à la paroi fixe augmente, et l'on peut éviter tout choc du tube contre la carcasse.

Par conséquent, un générateur de plus grande taille, et par suite d'énergie plus élevée, doit fournir des performances très intéressantes. Nous examinerons dans un prochain article [12] les possibilités d'extrapolation, à un niveau d'énergie de plus de $10 \mathrm{MJ}$, du dispositif coaxial expérimenté.

\section{Bibliographie}

[1] Sakharov, A. D. et al., Magnetic Cummulation, Sov. Phys. Dokl. 10 (1966) 1045.

[2] Sakharov, A. D., Magneto-Implosive Generators, Sov. Phys. Usp. 9 (1966) 294.

[3] Shearer, J. W. et al., Explosive-Driven Magnetic-Field Compression Generators, J. Appl. Phys. 39 (1968) 2102.

[4] Crawford, J. C. and Damerov, R. A., Explosively Driven High-Energy Generator. J. Appl. Phys. 39 (1968) 5224.

[5] KNoEPfel, H., KROEGLeR, H., LUPPI, R. et VAN MoNTFOORT, J., Generation and Switching of Magnetic Energies in the Mega-joule Range by Explosive Systems, Rev. Sci. Instrum. 40 (1969) 60.

[6] Antoni, B., Nazet, C., Pobe, L., Theoretical and Experimental Study of Explosive Driven Magnetic Field Compression Generators, International Conference on
Energy Storage, Compression and Switching, Turin, November, 4-7 (1974).

[7] Knoepfel, H., Kroegler, H., Luppi, R., Verbeex, R., Merits and Limitations of Explosively Driven Current Generators in Fusion Research. VI Symp. Fusion Technology. AACHEN (Sept. 1970).

[8] Hahn, R., Thèse d'Etat, Orsay (Avril 1975).

[9] Benort, A., Equilibrium Thermodynamic Data for the $\mathrm{H}_{2}-\mathrm{O}_{2}-\mathrm{He}$ System. UTIAS Technical Note $\mathrm{n}^{\circ} 128$ (August 1968).

[10] Aziz, A. K., Hurwitz, H. and Sternberg, H. M., Energy Transfer to a Rigid Piston under Detonation Loading, Phys. Fluids 4 (1961) 380.

[11] Antoni, B., Revue Phys. Appl. 9 (1974) 331.

[12] Hahn, R., Legentil, M., Rioux, C., soumis à Revue Phys. Appl. 